\title{
A Boosting Procedure for Variational-Based Image Restoration
}

\author{
Samad Wali ${ }^{1}$, Zhifang $\mathrm{Liu}^{1}$, Chunlin $\mathrm{Wu}^{1}$ and Huibin Chang ${ }^{2, *}$ \\ ${ }^{1}$ School of Mathematical Sciences, Nankai University, Tianjin, China \\ 2 School of Mathematical Sciences, Tianjin Normal University, Tianjin, China \\ Received 12 July 2017; Accepted (in revised version) 13 July 2017
}

\begin{abstract}
Variational methods are an important class of methods for general image restoration. Boosting technique has been shown capable of improving many image denoising algorithms. This paper discusses a boosting technique for general variational image restoration methods. It broadens the applications of boosting techniques to a wide range of image restoration problems, including not only denoising but also deblurring and inpainting. In particular, we combine the recent SOS technique with dynamic parameter to variational methods. The dynamic regularization parameter is motivated by Meyer's analysis on the ROF model. In each iteration of the boosting scheme, the variational model is solved by augmented Lagrangian method. The convergence analysis of the boosting process is shown in a special case of total variation image denoising with a "disk" input data. We have implemented our boosting technique for several image restoration problems such as denoising, inpainting and deblurring. The numerical results demonstrate promising improvement over standard variational restoration models such as total variation based models and higher order variational model as total generalized variation.
\end{abstract}

AMS subject classifications: 68U10, 90C25, 49M37

Key words: Variational method, image restoration, total variation, boosting, augmented Lagrangian method.

\section{Introduction}

Image restoration is the operation of estimating the clean or original image from an input corrupted image. These operations, such as denoising, inpainting and deblurring, are the most fundamental tasks in image processing. Suppose we have an observed image $f$, which is degraded from the true image $u$. In many cases the degradation can be expressed as follows:

$$
f=\mathscr{A} u+n,
$$

${ }^{*}$ Corresponding author. Email addresses: samad.walikhan@gmail . com (S. Wali), liuzhifang0628@163. com (Z. F. Liu), wucl@nankai.edu.cn (C. L. Wu), changhuibin@gmail.com (H. B. Chang) 
where $\mathscr{A}$ is a convolution operator and $n$ is some random noise such as Gaussian noise or impulsive noise. In order to estimate $u$, it is necessary to solve the above inverse and ill-posed problem.

To figure out an approximation $\hat{u}$ of the original image $u$, a large number of variational models and algorithms based energy regularization have been developed. One of the most successful regularization is the total variation regularization [38], which can preserve image edges quite well. Total variation has extensive applications and benefit effective optimization algorithms $[2,10,11,26,39,48,49,51,52]$. It also has been extended to higher order and vectorial models $[12,28,30,31,42,53,54]$ for gray scale and color image restoration. These models and algorithms rely on powerful image sparse representations $[6,19]$.

In spite of the performance and effectiveness of the above mentioned restoration algorithms, the result could be improved by applying a boosting technique. Boosting usually means a procedure calling an existing image processing algorithm iteratively, where the output of the current step is used as a part of input of the next step. This technique, to the best of our knowledge, has been only used in image denoising problem $(\mathscr{A}=I$ in Eq. (1.1)). We now review several existing boosting techniques. The "twicing method" [47] is very early and effective method which was proposed by Tukey. This method can be written as;

$$
\hat{u}_{k+1}=B(f)+B\left(f-\hat{u}_{k}\right),
$$

where $B(\cdot)$ represents the restoration algorithm and $\hat{u}_{k}$ is the $k$ th iteration of denoised image. This concept was used in [29] to improve filters. Another interesting earlier work was given in [43], where the authors have proposed an iterative procedure based on the ROF model [38]. The procedure generates a sequence $u_{k}$ which converges to the input image $f$. The procedure is stated as: $f=u^{\lambda}+v^{\lambda}$,

$$
\left[u^{\lambda}, v^{\lambda}\right]=\arg \min _{u+v=f} J\left(f, \lambda ; B V, L^{2}\right),
$$

where $\lambda$ is a weighting parameter serving as a scaling level to separate the two terms. This model was proposed for image decomposition based on hierarchical image representation of $f$. In [35] Osher et al proposed an iterative regularization method in which the residual was added back to the observed signal,

$$
\hat{u}_{k+1}=B\left(f+\sum_{i=1}^{k}\left(f-\hat{u}_{k}\right)\right) .
$$

In [17] the authors have proposed Unsharp Residual Iteration (URI) method which is given by,

$$
\hat{u}_{k+1}=\hat{u}_{1}+\left(\hat{u}_{k}-B\left(\hat{u}_{k}\right)\right) .
$$

This method was applied for texture, grant manipulation and transfer. Another similar approach was used in [16], which can be expressed as:

$$
\hat{u}_{k+1}=\hat{u}_{k}+B\left(f-\hat{u}_{k}\right) \text {. }
$$


A patch-based local boosting technique named as SAIF [46] was proposed to improve the denoising result. It has been shown that as $k$ increases, the estimate $\hat{u_{k}}$ returns to the noisy image $f$. The latest boosting method is the so called SOS boosting [37]. The improvement is achieved by repeating three simple steps (1) Strengthening the signal, (2) Operating the denoising algorithm, and (3) Subtracting the previous denoised image from the result. This procedure is as follows:

$$
\hat{u}_{k+1}=B\left(f+\hat{u}_{k}\right)-\hat{u}_{k} .
$$

This procedure is used for patch based image denoising methods NLM [7], K-SVD [20], BM3D [18] and EPLL [55]. The recursive function is initialized by setting $\hat{u_{0}}=0$. It has been shown quite effective for image denoising problem.

In this paper we combine the SOS boosting technique and variational image restoration method. We mention that, the combination of boosting technique with variational method can be used to solve general image restoration problems, including not only denoising but also deblurring and inpainting. We propose to use SOS with changing parameter to boost total variation based restoration models. The changing regularization parameter is motivated by Meyer's classical analysis [32] on the ROF model, which will be stated later. The proposed overall boosting scheme is as follows:

$$
\hat{u}_{k+1}=B\left(f+\hat{u}_{k}, \lambda_{k}\right)-\hat{u}_{k},
$$

where $\lambda_{k}$ is the regularization parameter at $k$ th iteration. We initialize $\lambda_{0}=\lambda$ which is the original model parameter.

The rest of the paper is organized as follows. In Section 2 we present ROF model and its developments, as well as the augmented Lagrangian method to solve the models. The Boosting algorithm and its motivation are discussed in Section 3. Section 4 discusses application of proposed algorithm, where we compare our results to the results by variational methods without boosting. A concluding remark and future direction are given in Section 5 .

\section{ROF model and its development}

Given an observed image $f: \Omega \rightarrow \mathbb{R}$ corrupted by the additive white Gaussian noise with zero mean, ROF model [38] is one of the basic variational models which is used for image restoration, i.e., to recover $u$ from $f$ in (1.1) by solving the following minimization problem:

$$
u^{\lambda}=\arg \min _{u}\|u\|_{B V(\Omega)}+\lambda\|f-u\|_{2}^{2},
$$

where $\Omega$ is bounded open subset of $\mathbb{R}^{2},\|\cdot\|_{B V(\Omega)}$ denotes total variation in the bounded variation (BV) space, $\|\cdot\|_{2}$ denotes the $L^{2}$ norm and $\lambda>0$ is a regularization parameter which balances the data-fidelity term against regularity term. The original input image is of size $n \times n$, i.e., $N=n^{2}$ pixels, and it is represented by a vector $u$ in lexicographical ordering. In this paper we study all the problems in discrete setting, where $\Omega$ denotes as 
an index set and $f \in \mathbb{R}^{N}$. The ROF model can be extended to a general minimization problem:

$$
u^{\lambda}=\arg \min _{u}\|\mathscr{W} u\|_{q}+\lambda\|f-\mathscr{A} u\|_{s}^{s},
$$

where $\mathscr{W}, \mathscr{A}$ are two linear operators and $\|\cdot\|_{q},\|\cdot\|_{s}$ with $0<q \leq 1,1 \leq s$ are two different type of norms existing in the regularization term and fidelity term respectively. For example, by setting $q=1, s=2$ and $\mathscr{W}=\nabla$ we obtain TV-L2 model (2.1) and we can also get high order model named as LLT model [30] with $\mathscr{W}=\nabla^{2}$. By setting $q=1, s=1$ and $\mathscr{W}=\nabla$, Eq. (2.2) is equal to TV-L1 model [50].

One can solve the above general minimization problem (2.2) for various image restoration tasks such as image denoising, deblurring and inpainting by setting different choice of linear operator $\mathscr{W}$ and fidelity terms. Next we give some examples in the following subsection.

\subsection{Image restoration}

\subsection{1. $\mathscr{A}=I$ for image denoising}

We elaborate on some examples related to image denoising if the images are corrupted by Gaussian noise or impulsive noises.

\section{- Gaussian noise}

In the case of input image corrupted by white Gaussian noise with zero mean, the ROF minimization problem has the following form in discrete setting:

$$
\min _{u} \operatorname{TV}(u)+\lambda\|f-u\|_{2}^{2}
$$

where the noisy image $f$ is rewritten as column vector $f \in \mathbb{R}^{N}$ using the lexicographical ordering, $\operatorname{TV}(u)$ is discrete version of total variation and defined as

$$
\operatorname{TV}(u)=\sum_{i=1}^{N} \sqrt{\left(\left[\nabla_{x} u\right]_{i}\right)^{2}+\left(\left[\nabla_{y} u\right]_{i}\right)^{2}}
$$

$\nabla$ is discrete gradient operator with periodic boundary conditions, $\left[\nabla_{x} u\right]_{i}$ and $\left[\nabla_{y} u\right]_{i}$ are the $x$-derivative and $y$-derivative located at the $i$-th pixel $(1 \leq i \leq N)$.

\section{- Impulsive noise}

If image is corrupted with non-Gaussian noise then the minimization problem has the following form:

$$
\min _{u} \operatorname{TV}(u)+\lambda\|f-u\|_{1} .
$$

The minimization problem (2.4) is suitable for recovering image corrupted by impulsive noise. 


\section{- High order TV case}

ROF model is well known to recover a signal or image with sharp edges. However, the restoration results have obvious staircase artifacts. In order to overcome these model dependent deficiencies, some variants of total variation by incorporating high order derivative have been proposed $[9,11-13,53],[42,54]$, which can reduce staircase artifacts effectively. In [30], Lysaker, Lundervold, and Tai proposed Hessian based TV model which is known as LLT model:

$$
\min _{u} H(u)+\lambda\|f-u\|_{2}^{2}
$$

where

$$
H(u)=\sum_{i=1}^{N} \sqrt{\left(\left[\nabla_{x x} u\right]_{i}\right)^{2}+\left(\left[\nabla_{x y} u\right]_{i}\right)^{2}+\left(\left[\nabla_{y x} u\right]_{i}\right)^{2}+\left(\left[\nabla_{y y} u\right]_{i}\right)^{2}} .
$$

Here $H(u)$ is the regularized term of higher order differential operator with periodic boundary conditions, and $\left[\nabla_{x x} u\right]_{i}$ and $\left[\nabla_{y y} u\right]_{i}$ are the second order $x$-derivative and $y$-derivative at the $i$-th pixel $(1 \leq i \leq N)$, and $\left[\nabla_{x y} u\right]_{i}$ and $\left[\nabla_{y x} u\right]_{i}$ are mixed second order derivatives.

More recently, one of the most popular high order TV methods was proposed by $[3,4]$, where the authors introduced total generalized variation (TGV) as the regularization term. For image denoising the model has following form

$$
\min _{u, v} \lambda_{1}\|\nabla u-v\|_{1}+\lambda_{2}\|\nabla \cdot v\|_{1}+\|f-u\|_{2}^{2},
$$

where $\lambda_{1}$ and $\lambda_{2}$ are regularization parameters, the variable $v=\left(v_{1}, v_{2}\right)$ varies in the space of all continuously differential 2 -tensors and

$$
\|\nabla \cdot v\|_{1}=\sum_{i=1}^{N} \sqrt{\left(\left[\nabla_{x} v_{1}\right]_{i}\right)^{2}+\left(\left[\nabla_{y} v_{1}\right]_{i}\right)^{2}+\left(\left[\nabla_{x} v_{2}\right]_{i}\right)^{2}+\left(\left[\nabla_{y} v_{2}\right]_{i}\right)^{2}} .
$$

Because the TGV regularizer is convex it allows to compute a globally optimal solution and it also improves ROF model (2.1).

\subsubsection{Image denoising and deblurring}

The degradation model for the blurred and noised images can be written as: $f=\mathscr{A} u+n$, where $f$ is an observed (degraded) image, $\mathscr{A}$ is the blur operator, $u$ is the clean image and $n$ denotes additive noise (often Gaussian). If we replace the $u$ in the unconstrained ROF model (2.3) with convolution $\mathscr{A} u$, then we arrive at TV deblurred model:

$$
\min _{u} \operatorname{TV}(u)+\lambda\|\mathscr{A} u-f\|_{2}^{2} .
$$

The minimization problem (2.7) has quadratic fidelity term (called TV-L2 model) is particularly suitable for recovering image corrupted by Gaussian noise and the blur at the 
same time. If image is blurred and corrupted with impulsive noise then the minimization problem has the following form as

$$
\min _{u} \operatorname{TV}(u)+\lambda\|\mathscr{A} u-f\|_{1},
$$

which is called TV-L1 model. As a result of such non-quadratic fidelity term in (2.8), it has great advantages in impulsive noise removal $[33,34]$ than the quadratic case.

\subsubsection{Image inpainting}

Image inpainting is the process of reconstructing lost or deteriorated parts of images. The given image $f$ is known only on the region $\Omega \backslash C$ and the task is to "interpolate" it to the whole region $\Omega$ by guessing the pixel values in region $C$. Inpainting by solving the total variation regularized model is an effective inpainting method which can be capable of recovering sharp edges, see the work for TV regularization applied to inapinting by Chan and Shen in [40]. Inpainting can be interpreted as image denoising with a spatially-varying regularization parameter [25]. TV problem can be written as follows

$$
\min _{u} \operatorname{TV}(u)+\lambda \sum_{i \in \Omega \backslash C}\left\|f_{i}-u_{i}\right\|_{2}^{2},
$$

where the parameter $\lambda>0$ to control the strength of the impact by total variation term. Readily one can see if setting $\lambda=0$, the known information $f$ is unused and $u$ is only influenced by the $\operatorname{TV}(u)$ term. Outside of index set $C$, the model perform TV-regularized denoising. Such kind of method can only deal with the case that the missing region is not a large continuous region. Otherwise, one needs the Euler's Elastica inpainting [41,44] and Exemplar-based inpainting by [1].

\subsection{Augmented Lagrangian method for solving TV based image restoration}

The numerical computation of TV based image restoration models suffers from difficulties due to its nonlinearity and non differentiability, the numerical algorithm in [38] is very slow as a result of the constraint about the time step in order to satisfy the stability condition $[14,15]$. Although there are many methods for the efficient solution of ROF model, we review one popular and important method, "augmented Lagrangian method (ALM)".

The augmented Lagrangian method is one of the classical methods which can solve constraint optimization problems $[27,36]$. It can be observed that $(2.2)$ is a more general optimization model. In this subsection we will focus on the specific cost function as ROF model, and briefly recall the ALM for solving it. For a general model (2.2), one can see details in Subsection 3.2.

The constrained problem for (2.3) as follows:

$$
\begin{aligned}
& \min _{u, p}\|p\|_{1}+\lambda\|f-u\|_{2}^{2}, \\
& \text { s.t. } \quad p=\nabla u .
\end{aligned}
$$




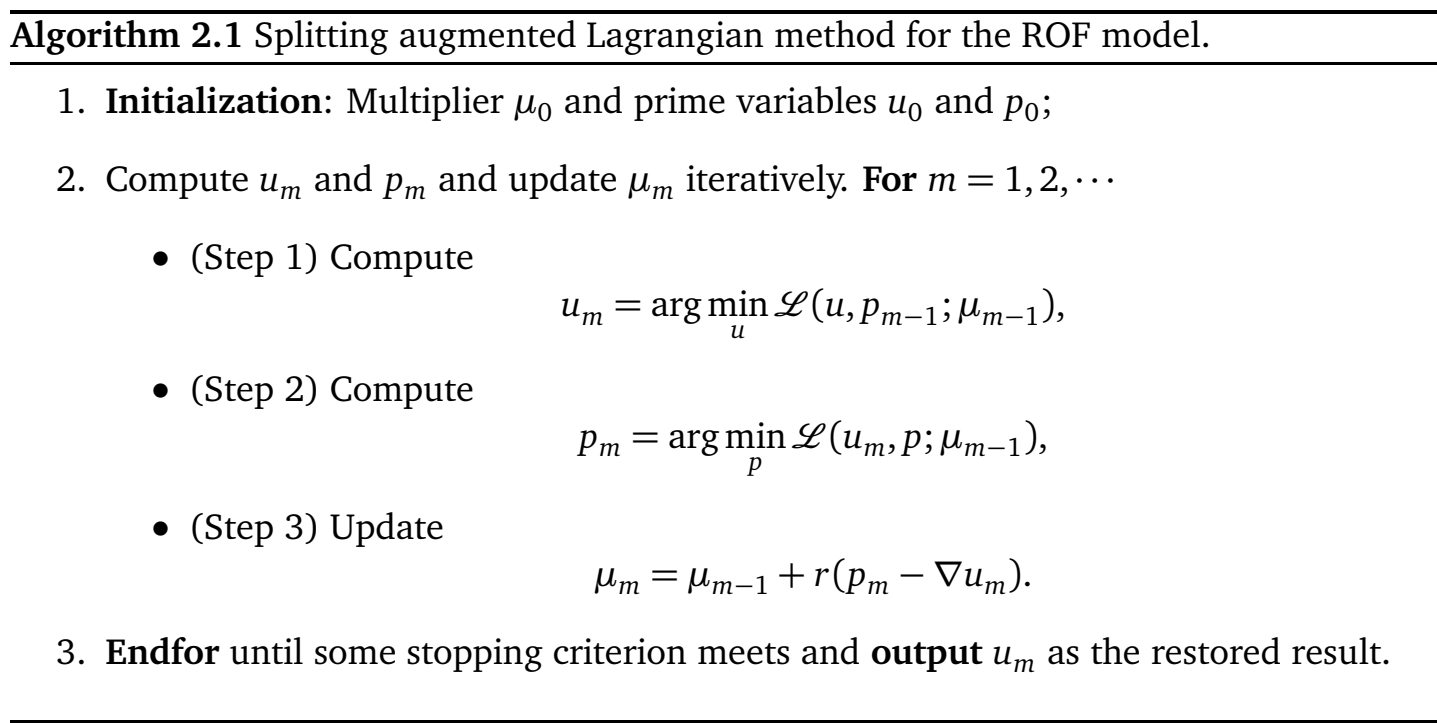

The augmented Lagrangian for constraint problem (2.10) can be obtained as follows:

$$
\mathscr{L}(u, p ; \mu)=\|p\|_{1}+\lambda\|f-u\|_{2}^{2}+\langle\mu, p-\nabla u\rangle+\frac{r}{2}\|p-\nabla u\|_{2}^{2},
$$

where $\mu$ is Lagrangian multiplier and $r$ is positive constant. We apply splitting version of ALM to solve the above saddle-point problem corresponding to the augmented Lagrangian functional (2.11), please see Algorithm 2.1.

The classical augmented Lagrangian method can solve the solution of original problem (2.11) by minimizing the subproblems $u_{m}$ and $p_{m}$ in coupled together. But subproblems are usually not readily to be solved. Therefore, one can compute the variable $u_{m}$ and $p_{m}$ using an alternative minimization procedure as a Gauss-Seidel style and therefore a splitting ALM can be designed without inner loops for coupled subproblems, see Refs. [26, 48, 49] for details.

\section{Boosting procedure}

In this section we elaborate on our main idea, motivations, the proposed algorithm and corresponding convergence discussion.

\subsection{Main idea and its motivation}

The word "boosting" is derived from Machine Learning and it means to produce a strong learner through a combination of weak learners (see [21] for more details). However, in this paper boosting refers to improve the performance of restoration process iteratively, where we take variational method as a "Black Box" (means tool).

The variational problems have space of improvement in image processing. The purpose of boosting in this paper is to improve TV related variational methods further, and 


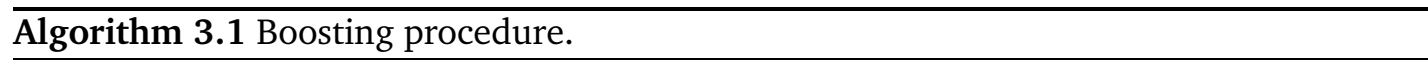

1. Initialization:

- $\hat{u}_{0}=0, f_{0}=f$ and parameter $\lambda_{0}=\lambda$,

- Set $\rho>0$ and $\tau>1$.

2. For $k=1,2, \cdots$;

- (Step 1) Strengthening image and tuning parameter computation: Update

$$
f_{k}=f_{0}+\rho \hat{u}_{k} \& \lambda_{k}=\tau^{k} \lambda,
$$

- (Step 2) Image restoration: Solve

$$
u_{k}=\arg \min _{u}\|\mathscr{W} u\|_{q}+\lambda_{k}\left\|f_{k}-\mathscr{A} u\right\|_{s}^{s},
$$

- (Step 3) Deduction: Update

$$
\hat{u}_{k+1}=u_{k}-\rho \hat{u}_{k} .
$$

3. Endfor until some stopping criterion meets and output $\hat{u}_{k+1}$ as the boosting result.

increase the quality of restored results. Our idea in this paper is more inspired by the SOS technique [37]. In this work, we extend the idea of [37] to restore corrupted images by variational method. We will give a theoretical discussion on the differences between our new boosting method and SOS boosting by analyzing a special function in the following subsections. Here our apparently novel idea is to replace the variation problem (2.2) by a sequence (1.8), so as to obtain an improved restored image, for a wide class of inverse imaging problems. The output sequence $\left\{\hat{u}_{k+1}\right\}$ by our method has two terms; first one is $B\left(f+\hat{u}_{k}, \lambda_{k}\right)$, where $B(\cdot)$ is a nonlinear operator which plays a role of restoration with updating $\lambda$. The second term performs subtraction of denoised image and the purpose of subtraction is to show restored image more close to the reference or original image. In our method the variable $f$ and parameter $\lambda$ change each time in the following way, $f_{\text {new }} \leftarrow f_{\text {original }}+u^{\lambda_{\text {old }}}, \quad \lambda_{\text {new }} \leftarrow \tau \lambda_{\text {old }}$. In other words, we take the solution from the previous step and apply the restoration algorithm $B(\cdot)$. With $\lambda_{k}=\lambda_{0} \tau^{k}$, we obtain our final result by outputting $\left\{\hat{u}_{k+1}\right\}$ after $k$ steps. We proposed the boosting procedure in Algorithm 3.1 for the mentioned general TV based variation model as follows.

\subsection{Solve subproblems of (3.2)}

In this subsection we solve the subproblems of our boosting Algorithm 3.1 by ALM. 
The energy functional for general minimization problem (2.2) can be written as follows

$$
\min _{u}\{E(u)=G(\mathscr{W} u)+H(\mathscr{A} u, f)\}
$$

where $G(\mathscr{W} u)=\|\mathscr{W} u\|_{q}$ and $H(\mathscr{A} u, f)=\lambda\|f-\mathscr{A} u\|_{s}^{s}$. The constraint minimization problem for (3.4) reads as follows

$$
\begin{aligned}
& \min _{u, p, z} G(p)+H(z, f) m \\
& \text { s.t. } \quad p=\mathscr{W} u, \quad z=\mathscr{A} u .
\end{aligned}
$$

The augmented Lagrangian for constraint problem (3.5):

$$
\begin{aligned}
& \mathscr{L}\left(u, p, z ; \mu^{1}, \mu^{2}\right) \\
= & G(p)+H(z, f)+\left\langle\mu^{1}, p-\mathscr{W} u\right\rangle+\left\langle\mu^{2}, z-\mathscr{A} u\right\rangle+\frac{r^{1}}{2}\|p-\mathscr{W} u\|_{2}^{2}+\frac{r^{2}}{2}\|z-\mathscr{A} u\|_{2}^{2},
\end{aligned}
$$

where $\mu^{1}$ and $\mu^{2}$ are the Lagrangian multipliers and $r^{1}$ and $r^{2}$ are the positive parameters. We apply an splitting ALM algorithm or alternating direction method of multipliers to solve the above saddle-point problem corresponding to the augmented Lagrangian(3.6), and see Algorithm 3.2

In the following we show how to efficiently solve these sub-problems and then present an alternative minimization algorithm to solve.

\section{- Solution of $u$-subproblem}

The problem (3.7) is a quadratic optimization problem, whose optimality condition reads

$$
\mathscr{W}^{*} \mu_{m}^{1}+\mathscr{A}^{*} \mu_{m}^{2}+r^{1} \mathscr{W}^{*}(p-\mathscr{W} u)+r^{2} \mathscr{A}^{*}(z-\mathscr{A} u)=0,
$$

and the related gradient operator is discretized using periodic boundary conditions. Following $[45,48,49,51,52]$, we use Fourier transform with an FFT implementation. Denoting $\mathscr{F}(\cdot)$ as the Fourier transform, we write the solution as follows:

$$
u=\mathscr{F}^{-1}\left(\frac{\mathscr{F}\left(\mathscr{W}^{*}\right) \mathscr{F}\left(\mu_{m}^{1}+r^{1} p\right)+\mathscr{F}\left(\mathscr{A}^{*}\right) \mathscr{F}\left(\mu_{m}^{2}+r^{2} z\right)}{r^{1} \mathscr{F}\left(\mathscr{W}^{*}\right) \mathscr{F}(\mathscr{W})+r^{2} \mathscr{F}\left(\mathscr{A}^{*}\right) \mathscr{F}(\mathscr{A})}\right),
$$

where $\mu_{m}^{1}=\left(\mu_{m}^{1 x}, \mu_{m}^{1 y}\right)$ and $p=\left(p^{x}, p^{y}\right)$ and the division $\div$ denotes pointwise operator. The Fourier transform of the operators $\mathscr{A}$ and $\mathscr{W}$ are regarded as the transforms of their corresponding convolution kernels.

\section{- Solution of $p$-sub problem}

When $q=1$, (3.8) has the following closed form denoting by the soft thresholding as

$$
p=\operatorname{Thresh}_{1 / r^{1}}(w),
$$

where $\operatorname{Thresh}_{\eta}(w):=\max \{0,|w|-\eta\} \operatorname{sign}(w)$, and

$$
w=\mathscr{W} u-\frac{\mu_{m}^{1}}{r^{1}} .
$$


$\overline{\text { Algorithm 3.2 Splitting augmented Lagrangian method for the general minimization mod- }}$ el (2.2).

1. Initialization: Multipliers $\mu_{0}^{1}, \mu_{0}^{2}$ and prime variables $u_{0}, p_{0}, z_{0}$;

2. Compute $u_{m}, p_{m}$ and $z_{m}$ and update $\mu_{m}^{1}$ and $\mu_{m}^{2}$ iteratively. For $m=1,2, \cdots$

- (Step 1) Compute

$$
u_{m}=\arg \min _{u} \mathscr{L}\left(u, p_{m-1}, z_{m-1} ; \mu_{m-1}^{1}, \mu_{m-1}^{2}\right),
$$

- (Step 2) Compute

$$
p_{m}=\arg \min _{p} \mathscr{L}\left(u_{m}, p, z_{m-1} ; \mu_{m-1}^{1}, \mu_{m-1}^{2}\right),
$$

- (Step 3) Compute

$$
z_{m}=\arg \min _{z} \mathscr{L}\left(u_{m}, p_{m}, z ; \mu_{m-1}^{1}, \mu_{m-1}^{2}\right)
$$

- (Step 4) Update

$$
\begin{aligned}
& \mu_{m}^{1}=\mu_{m-1}^{1}+r^{1}\left(p_{m}-\mathscr{W} u_{m}\right), \\
& \mu_{m}^{2}=\mu_{m-1}^{2}+r^{2}\left(z_{m}-\mathscr{A} u_{m}\right) .
\end{aligned}
$$

3. Endfor of $m$ until some stopping rule meets and output $u_{m}$ as the restored result.

\section{- Solution of z-subproblem}

For $s=2, z$-subproblem (3.9) becomes

$$
\min _{z} \lambda\|f-z\|_{2}^{2}+\left\langle\mu_{m}^{2}, z\right\rangle+\frac{r^{2}}{2}\|z-\mathscr{A} u\|_{2}^{2}
$$

The Euler Lagrangian equation for (3.11) is

$$
\left(2 \lambda+r^{2}\right) z=2 \lambda f-\mu_{m}^{2}+r^{2} \mathscr{A} u,
$$

which has closed form solution i.e.,

$$
z=\frac{2 \lambda f-\mu_{m}^{2}+r^{2} \mathscr{A} u}{2 \lambda+r^{2}} .
$$

For $s=1, z$-subproblem (3.9) becomes

$$
\min _{z} \lambda\|f-z\|_{1}+\left\langle\mu_{m}^{2}, z\right\rangle+\frac{r^{2}}{2}\|z-\mathscr{A} u\|_{2}^{2},
$$


which has closed form solution i.e.,

$$
z_{i}=f_{i}+\max \left(0,1-\frac{\lambda}{r^{2}\left|w_{i}-f_{i}\right|}\right)\left(w_{i}-f_{i}\right) \quad \forall i
$$

where

$$
w=\mathscr{A} u-\frac{\mu_{m}^{z}}{r^{2}}
$$

\subsection{Convergence analysis for noise free data in continuous setting}

In this subsection we show some discussion on the convergence of our boosting method. It seems difficult for us to study the convergence for our boosting method. However, we can give some analysis based on a special but important function for $f$ following the work in ([32], pp. 36), where Meyer analyzed the property of the solution of ROF minimization for a noise free data. Note that our following analysis is present in the continuous setting for our proposed boosting method defined over the region $\Omega:=\mathbb{R}^{2}$.

Lemma 3.1. ([32])Suppose $f$ is a function such that $f=\beta I_{R}(x)$, where $\beta$ is a positive constant and $I_{R}(x)$ is an indicator function defined as

$$
I_{R}(x)= \begin{cases}1, & \text { when }|x| \leq R, \\ 0, & \text { otherwise, }\end{cases}
$$

the solution of ROF model (2.1) can be expressed as

$$
u^{\lambda}= \begin{cases}\left(\beta-\frac{1}{\lambda R}\right) I_{R}(x), & \text { when } \lambda \beta R \geq 1 \\ 0, & \text { otherwise. }\end{cases}
$$

Following the setting for the above lemma, we can give the convergence result for a special noise-free measurement $f$ as follows.

Theorem 3.1. Assume that $f$ is an observed noise free data defined as: $f=\beta I_{R}(x)$ and suppose the minimization problem of ROF model is exactly solved in each iteration. If the parameters in our boosting method are set as $\tau>1$ and $\rho>0$, then the sequence $\left\{\hat{u}_{k+1}\right\}$ generated by new boosting algorithm satisfies

$$
\lim _{k \rightarrow \infty} \hat{u}_{k+1}=f .
$$

Proof. We first proof the following sequence by mathematical induction, which is generated from our boosting method by using the Meyer's classical example.

$$
\hat{u}_{k+1}=\left(\beta-\frac{1}{\tau^{k} \lambda R}\right) I_{R}(x), \text { if } \tau^{k} \lambda R\left(\beta(\rho+1)-\frac{\rho}{\tau^{k-1} \lambda R}\right) \geq 1 .
$$


According to our boosting algorithm, for $k=0, \hat{u}_{0}=0, f_{0}=f+\rho \hat{u}_{0}=f$ and $\lambda_{0}=\tau^{0} \lambda$,

$$
\begin{aligned}
& u_{0}=u^{\lambda}=\left(\beta-\frac{1}{\lambda R}\right) I_{R(x)}, \\
& \hat{u}_{1}=u_{0}-\rho \hat{u}_{0}=u_{0} .
\end{aligned}
$$

For $k=1, f_{1}=f_{0}+\rho \hat{u}_{1}$, and $\lambda_{1}=\tau \lambda_{0}$, which shows that for new observed value $f_{1}, \beta$ becomes $\left(\beta(1+\rho)-\frac{\rho}{\lambda R}\right)$ and regularization parameter of model is $\tau \lambda$ and satisfy that:

$$
\tau \lambda R\left(\beta(1+\rho)-\frac{\rho}{\lambda R}\right) \geq 1
$$

The ROF minimization problem can be written as:

$$
u_{1}=\arg \min _{u}\left\{\|u\|_{B V(\Omega)}+\lambda_{1}\left\|f_{1}-u\right\|_{2}^{2}\right\} .
$$

The solution according to Meyer's approach is given by,

$$
\begin{aligned}
& u_{1}=\left(\beta(1+\rho)-\frac{1}{\lambda R}\left(\rho+\frac{1}{\tau}\right)\right) I_{R}(x), \\
& \hat{u}_{2}=u_{1}-\rho \hat{u}_{1}=\left(\beta-\frac{1}{\tau \lambda R}\right) I_{R}(x) .
\end{aligned}
$$

Assume that (3.14) is true for some $k=n-1 \geq 1$, i.e.,

$$
\hat{u}_{n}=\left(\beta-\frac{1}{\tau^{n-1} \lambda R}\right) I_{R}(x),
$$

we prove (3.14) is true for $k=n$. For $k=n$,

$$
f_{n}=f+\rho \hat{u}_{n}=\left(\beta(1+\rho)-\frac{\rho}{\tau^{n-1} \lambda R}\right) I_{R}(x),
$$

this scheme satisfy the following general condition:

$$
\begin{aligned}
\beta \lambda R & \geq \tau \lambda R\left(\beta(1+\rho)-\frac{\rho}{\lambda R}\right) \geq \tau^{2} \lambda R\left(\beta(1+\rho)-\frac{\rho}{\tau \lambda R}\right) \\
& \geq \cdots \geq \tau^{n} \lambda R\left(\beta(1+\rho)-\frac{\rho}{\tau^{n-1} \lambda R}\right) \geq 1 .
\end{aligned}
$$

The solution for (3.15) is

$$
u_{n}=\left(\beta(1+\rho)-\frac{1}{\tau^{n-1} \lambda R}\left(\rho+\frac{1}{\tau}\right)\right) I_{R}(x),
$$

and our boosting can be compute as,

$$
\hat{u}_{n+1}=u_{n}-\rho \hat{u}_{n}=\left(\beta-\frac{1}{\tau^{n} \lambda R}\right) I_{R}(x),
$$


its corresponding minimization problem would be as:

$$
u_{n}=\arg \min _{u}\left\{\|u\|_{B V(\Omega)}+\lambda_{n}\left\|f_{n}-u\right\|_{2}^{2}\right\}
$$

where $\lambda_{n}=\tau^{n} \lambda$.

By taking limit as $k \rightarrow \infty$ on (3.14), we have

$$
\lim _{k \rightarrow \infty} \hat{u}_{k+1}=\beta I_{R}(x)=f,
$$

which completes the proof.

Remark 3.1. Under the same assumptions, the sequence $\left\{\hat{u}_{k}\right\}$ obtained directly from the SOS boosting technique [37] is not convergent for variational problem, that can be proved by dividing SOS technique into three steps for $k=0,1$ as follows.

As $k=0, \hat{u}_{0}=0$ and $f_{0}=f+\rho \hat{u}_{0}$ the minimization problem can be denoted as

$$
u_{0}=\arg \min _{u}\left\{\|u\|_{B V(\Omega)}+\lambda\left\|f_{0}-u\right\|_{2}^{2}\right\} .
$$

The solution is readily obtained by Meyer's approach as

$$
u_{0}=\left(\beta-\frac{1}{\lambda R}\right) I_{R(x)}, \quad \hat{u}_{1}=u_{0}-\rho \hat{u}_{0}=u_{0} .
$$

For $k=1$, we have

$$
f_{1}=f_{0}+\rho \hat{u}_{1}=\left(\beta(1+\rho)-\frac{\rho}{\lambda R}\right) I_{R(x)},
$$

which satisfies the positivity property

$$
\lambda R\left(\beta(1+\rho)-\frac{\rho}{\lambda R}\right) \geq 1 .
$$

The following minimization problem can be written as

$$
u_{1}=\arg \min _{u}\left\{\|u\|_{B V(\Omega)}+\lambda\left\|f_{1}-u\right\|_{2}^{2}\right\},
$$

and the corresponding solution by Meyer's approach is given as

$$
u_{1}=\left(\beta(1+\rho)-\frac{\rho}{\lambda R}-\frac{1}{\lambda R}\right) I_{R(x)}=(1+\rho)\left(\beta-\frac{1}{\lambda R}\right) I_{R(x)} .
$$

Finally we have

$$
\hat{u}_{2}=u_{1}-\rho \hat{u}_{1}=(1+\rho)\left(\beta-\frac{1}{\lambda R}\right) I_{R(x)}-\rho\left(\beta-\frac{1}{\lambda R}\right) I_{R(x)}=\left(\beta-\frac{1}{\lambda R}\right) I_{R(x)}=\hat{u}_{1} .
$$

It implies that we get the same value as the previous iteration, and therefore we conclude that $\lim _{k \rightarrow \infty} \hat{u}_{k+1} \neq f$. 
Remark 3.2. The above theorem is established for noise free data, and by such approach we can compute the solution and its convergence to a special type of observed signal $f$. It will be more interesting to consider the case with noisy measurement $f$. However it seems that such above approach can not work, and we leave the challengeable task as a future work.

The very recent and related work [37] adopted a procedure named SOS used for local and patch based image denoising method. The main differences between our proposed method and SOS method are stated in three aspects. First In our proposed method the regularization parameter is adaptive which varies in each iteration. Specifically, the value of $\lambda_{k}$ at $k_{t h}$ step is updated as $\lambda_{k}=\tau^{k} \lambda$, and see details in the above boosting procedure. Second, when $\tau=1$ our method is similar to SOS technique and with this condition the signal could be slightly improved but not always, especially for image deblurring. Last, we implemented our model to variational based image restoration, while SOS technique is only considered for patch based image denoising methods.

\section{Numerical experiments}

In this section, we will provide several applications by the proposed method, namely for image denoising, deblurring, and inpainting. Eight images in Fig. 1 as the ground truth are tested. All the experiments were conducted with MATLAB R2013b on a HP Z228 desktop with Intel(R) Core(TM) i7-4790 CPU @3.60GHz and 8GB memory. The performance is evaluated by the signal to noise ratio (SNR) and this quality metric for boosting is defined

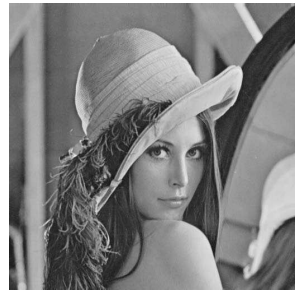

(a) Lena. Size: $512 \times$ 512

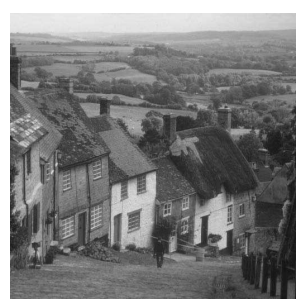

(e) Hill. Size: $512 \times$ 512

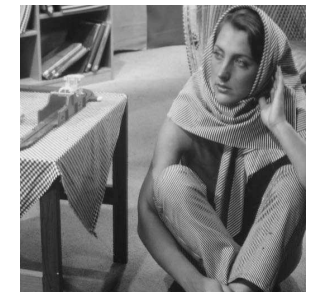

(b) Barbara. Size:512 $\times 512$

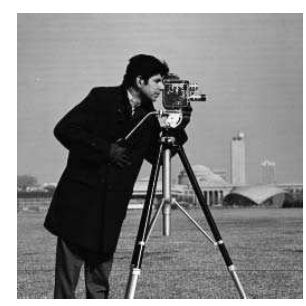

(f) Cameraman. Size: $256 \times 256$

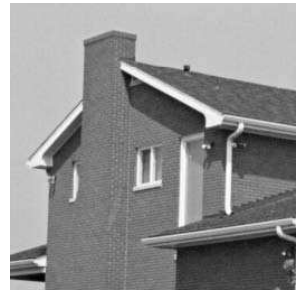

(c) House. Size: 256 $\times 256$

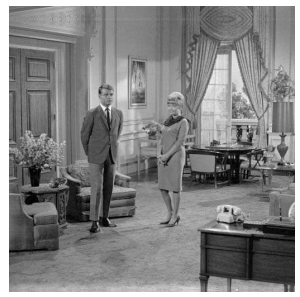

(g) Couple. Size: 512 $\times 512$

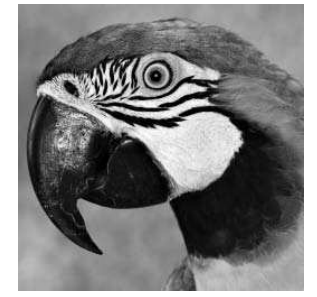

(d) Parrot. Size: 256 $\times 256$

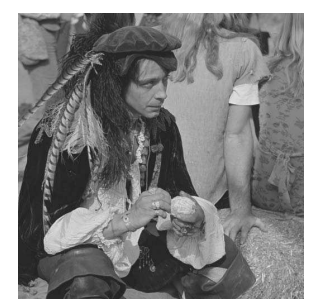

(h) Man. Size: $512 \times$ 512

Figure 1: Test Images used in this paper. 
as:

$$
\mathrm{SNR}_{k} \triangleq 10 \log _{10} \frac{\|u-\bar{u}\|^{2}}{\left\|u-\hat{u}_{k+1}\right\|^{2}}
$$

where $u$ is the original signal, $\bar{u}$ is the mean intensity value of $u$ and $\hat{u}_{k+1}$ is boosted signal.

\subsection{Comparing with other two boosting methods}

To show the advantage of our proposed method with other existing methods, Fig. 2 shows the differences between our proposed method and other two boosting techniques including SOS and Osher's iterative regularization method (1.4). Readily one can infer that the three boosting methods restored signals with higher quality than TV. Visually our proposed method and Osher's outperforms SOS. Although the SNRs and MSEs are very close for ours and Osher's method, the image quality is higher visually than that by Osher's method. Therefore, in the following tests we only compare our method with TV.

\subsection{Image denoising}

The regularization parameter $\lambda$ plays a central role in our experiments. The selection of $\lambda$ affects the balance between noise removal and signal structure preservation. One simple way for parameter tuning is the discrepancy principle [22], where $\lambda$ is selected to match the noise variance $\sigma^{2}$. For TV denoising one solved a constrained form of ROF problem

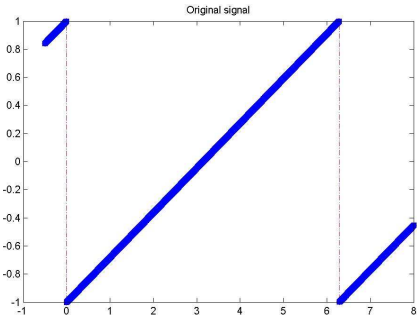

(a)

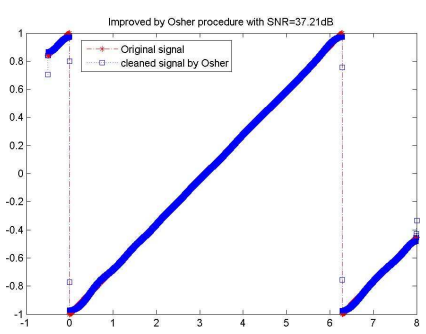

(d)

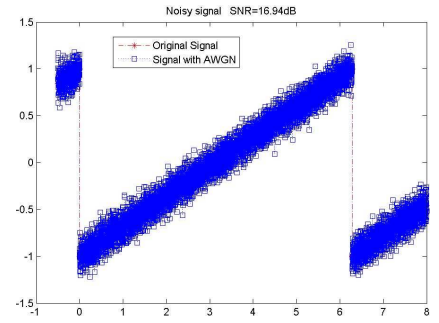

(b)

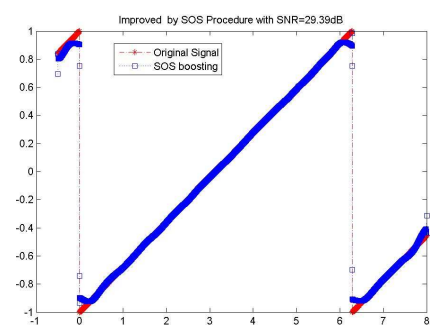

(e)

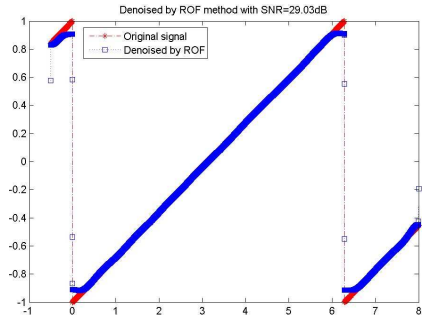

(c)

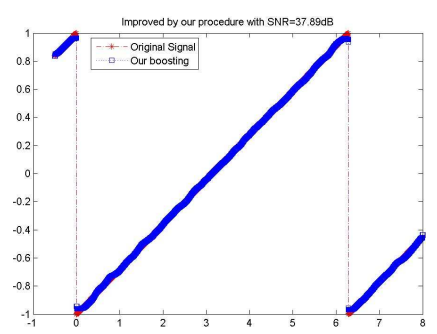

(f)

Figure 2: (a) is original signal, (b) is noisy signal by AWGN with SNR value $17 \mathrm{~dB},(\mathrm{c}),(\mathrm{e})$ are denoised signals by ROF-TV model, and by SOS boosting procedure respectively with $\lambda=0.05$, (d) is cleaned signal by Osher's iterative regularization method [35] with $\lambda=0.02$ and ( $f$ ) is recovered by our boosting procedure with $\lambda_{0}=0.9, \rho=0.015, \tau=1.212$ for $k=6$. 


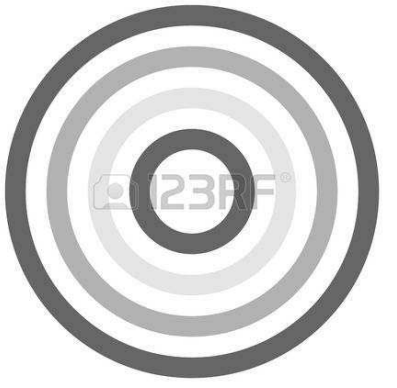

(a) Original image

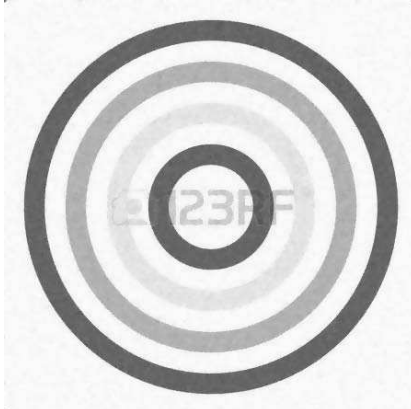

(d) TV-Boost, SNR: 18.89

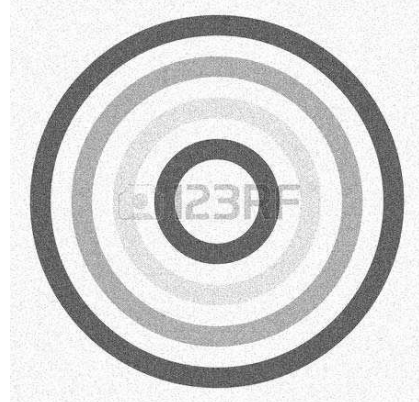

(b) Noisy, SNR: 13.32

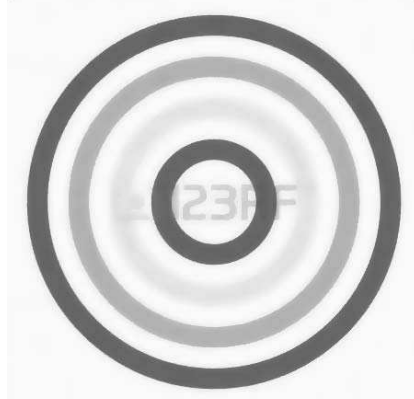

(e) Denoised TGV, SNR:17.83

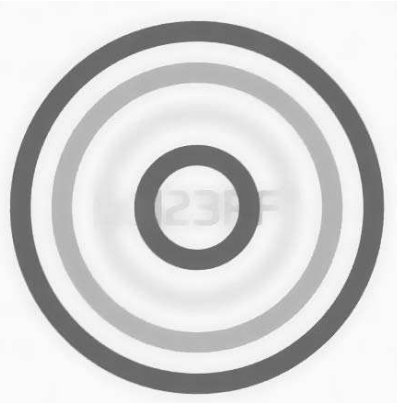

(c) Denoised TV, SNR: 16.20

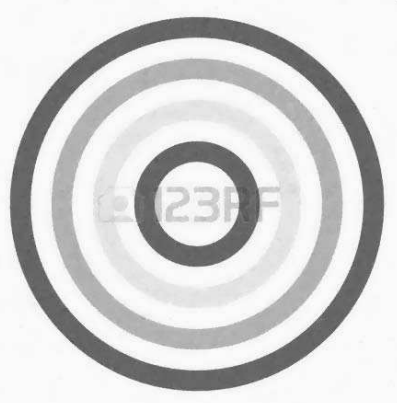

(f) TGV-Boost, SNR: 19.05

Figure 3: image denoising. (a): original image, (b): image corrupted by random Gaussian noise $\sigma=15$, (c): denoised image by TV algorithm, (d): TV boosting image by our method with $\lambda_{0}=5.2140, \rho=0.66$ and $\tau=1.22$ for $k=7,(\mathrm{e})$ : denoised image by TGV algorithm, (f): TGV boosting image by our method with $\lambda_{0}=0.09, \rho=1.9$ and $\tau=0.8$ for $k=3$.

(2.1). Indeed, in [8] Chambolle proposed an effective algorithm to find the optimal $\lambda$ so that $\|f-u\|_{2}^{2} \simeq \sigma^{2}$, where one can compute a unique $\lambda$ satisfying $\|f-u\|_{2}^{2}=\sigma^{2}$. In [23], the author initialized the parameter $\lambda_{\text {int }}$ to obtain the unique value of $\lambda$ by the following empirical formula

$$
\lambda_{\text {int }}=\frac{0.7079}{\sigma}+\frac{0.6849}{\sigma^{2}}
$$

where $\sigma$ is the standard deviation. For TV denoising we perform the experiment with $\lambda$ computed by the empirical formula, while for our boosting method we use the same $\lambda$ as the initial guess, and select other boosting parameters $\rho$ and $\tau$ empirically. In the experiments set $k=3$ for the outer loop. The algorithm in the inner iteration of our algorithm is used the same as for TV denoising model.

In this experiment, we evaluate the improved denoising results of TV and TGV [4] by applying our boosting procedure. The TGV is a higher order model which can improves the TV model. We first tests our method on a synthetic image in Fig. 3(a). In Fig. 3(c) one can observe that TV model reduces noise but at the same time it smooths away small structures while Fig. 3(d) shows the improvement of TV model by applying our boosting procedure. The TGV has better results but also slightly smooths some parts of image, the 
Table 1: Comparison between the image denoising results (SNR in $\mathrm{dB}$ ) of TV and TGV algorithms and their boosting outcomes.

\begin{tabular}{|c|c|c|c|c|c|c|c|c|}
\hline$\sigma$ & Image & $\lambda_{0}$ & $\rho$ & $\tau$ & TV & TV-Boost & TGV & TGV-Boost \\
\hline \multirow{6}{*}{10} & Man & 23.23 & 0.1 & 1.3 & 17.82 & 19.46 & 17.99 & 19.51 \\
\hline & Cameraman & 24.41 & 0.4 & 1.24 & 18.96 & 20.40 & 19.22 & 20.52 \\
\hline & House & 19.80 & 0.4 & 1.21 & 18.29 & 20.41 & 18.56 & 19.51 \\
\hline & Parrot & 22.84 & 0.53 & 1.24 & 19.30 & 20.81 & 19.70 & 20.90 \\
\hline & Barbara & 31.58 & 0.31 & 1.25 & 15.38 & 16.67 & 15.63 & 16.77 \\
\hline & Lena & 19.22 & 0.43 & 1.23 & 18.27 & 19.63 & 18.78 & 19.88 \\
\hline \multirow{6}{*}{15} & Man & 14.45 & 0.2 & 1.26 & 15.76 & 17.24 & 15.96 & 17.30 \\
\hline & Cameraman & 15.11 & 0.42 & 1.23 & 16.71 & 18.09 & 17.06 & 18.20 \\
\hline & House & 12.47 & 0.42 & 1.2 & 16.56 & 17.49 & 16.90 & 17.50 \\
\hline & Parrot & 14.11 & 0.58 & 1.24 & 17.04 & 18.48 & 17.51 & 18.57 \\
\hline & Barbara & 20.19 & 0.35 & 1.19 & 13.17 & 14.15 & 13.40 & 14.20 \\
\hline & Lena & 13.02 & 0.39 & 1.19 & 16.68 & 17.74 & 16.92 & 18.05 \\
\hline \multirow{6}{*}{20} & Man & 9.91 & 0.3 & 1.25 & 14.19 & 15.74 & 14.63 & 15.77 \\
\hline & Cameraman & 10.50 & 0.43 & 1.23 & 15.09 & 16.46 & 15.40 & 16.57 \\
\hline & House & 9.32 & 0.43 & 1.17 & 15.44 & 16.28 & 15.81 & 16.32 \\
\hline & Parrot & 9.85 & 0.57 & 1.25 & 15.40 & 16.94 & 15.87 & 17.03 \\
\hline & Barbara & 12.98 & 0.37 & 1.21 & 11.84 & 12.65 & 12.06 & 12.61 \\
\hline & Lena & 9.49 & 0.4 & 1.17 & 15.47 & 16.53 & 15.64 & 16.81 \\
\hline \multirow{6}{*}{25} & Man & 7.24 & 0.2 & 1.28 & 12.92 & 14.57 & 13.82 & 14.64 \\
\hline & Cameraman & 7.8612 & 0.48 & 1.22 & 13.72 & 15.22 & 14.49 & 15.23 \\
\hline & House & 7.53 & 0.48 & 1.15 & 14.62 & 15.42 & 13.92 & 15.51 \\
\hline & Parrot & 7.50 & 0.61 & 1.21 & 13.95 & 15.61 & 14.93 & 15.68 \\
\hline & Barbara & 9.79 & 0.41 & 1.20 & 10.89 & 11.69 & 11.13 & 11.62 \\
\hline & Lena & 7.38 & 0.41 & 1.18 & 14.52 & 15.56 & 14.90 & 15.77 \\
\hline \multirow{6}{*}{30} & Man & 5.59 & 0.1 & 1.3 & 11.87 & 13.63 & 12.25 & 13.63 \\
\hline & Cameraman & 6.1758 & 0.51 & 1.25 & 12.73 & 14.27 & 12.85 & 14.33 \\
\hline & House & 6.04 & 0.51 & 1.15 & 13.61 & 14.57 & 13.07 & 14.64 \\
\hline & Parrot & 5.79 & 0.59 & 1.28 & 12.78 & 14.64 & 13.19 & 14.70 \\
\hline & Barbara & 7.27 & 0.45 & 1.21 & 10.20 & 10.98 & 10.35 & 10.95 \\
\hline & Lena & 6.21 & 0.41 & 1.16 & 13.69 & 14.81 & 14.29 & 14.93 \\
\hline
\end{tabular}

Table 2: Comparison between the image deblurring results (SNR in $\mathrm{dB}$ ) of TV algorithm and its boosting outcomes.

\begin{tabular}{||c|c|c|cc|cc|cc|cc|cc||}
\hline$r$ & \multirow{2}{*}{$\lambda$} & \multirow{2}{*}{$\tau$} & \multicolumn{2}{|c|}{ Lena } & \multicolumn{2}{c|}{ Parrot } & \multicolumn{2}{c|}{ Couple } & \multicolumn{2}{c||}{ Cameraman } & \multicolumn{2}{c||}{ House } \\
\cline { 4 - 29 } & & & Deblur & Boost & Deblur & Boost & Deblur & Boost & Deblur & Boost & Deblur & Boost \\
\hline 4 & 769.81 & 1.6 & 20.46 & 23.75 & 18.45 & 22.50 & 16.58 & 21.13 & 17.64 & 22.27 & 20.04 & 25.41 \\
\hline 6 & 1154.7 & 1.7 & 18.26 & 21.71 & 16.31 & 20.31 & 14.64 & 19.22 & 15.59 & 20.18 & 18.86 & 22.91 \\
\hline 8 & 1539.62 & 1.8 & 16.92 & 20.29 & 16.69 & 18.64 & 13.42 & 17.69 & 14.34 & 18.56 & 17.89 & 20.93 \\
\hline 10 & 1924.52 & 1.9 & 15.96 & 19.17 & 13.03 & 17.30 & 12.36 & 16.29 & 13.20 & 17.10 & 16.70 & 19.71 \\
\hline 12 & 2309.4 & 2 & 15.06 & 18.10 & 12.36 & 16.22 & 11.47 & 14.90 & 12.56 & 15.94 & 15.50 & 18.07 \\
\hline
\end{tabular}




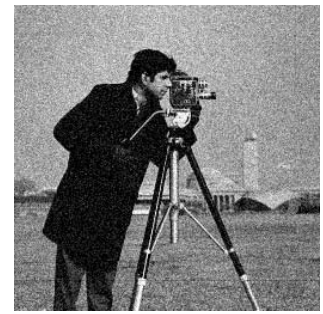

(a)

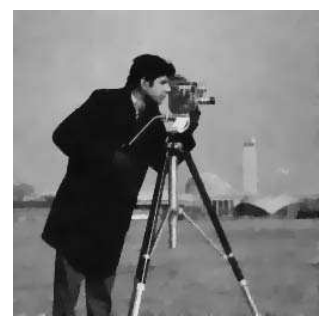

(e)

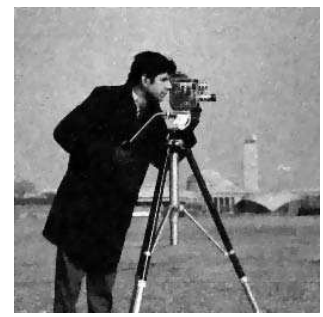

(i)

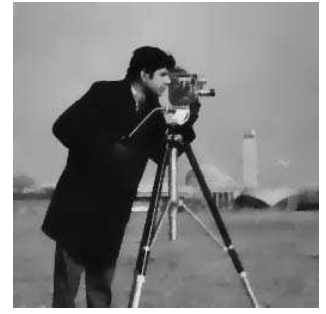

(m)

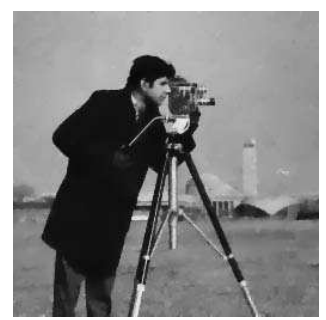

(q)

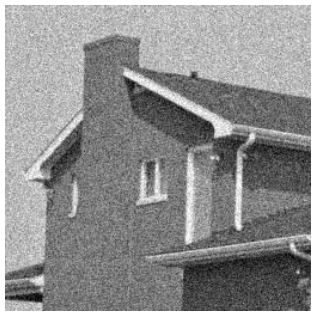

(b)

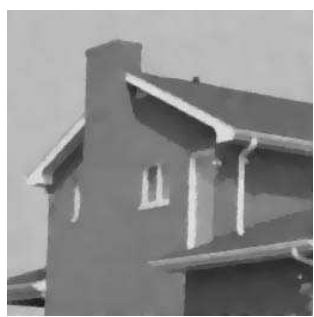

(f)

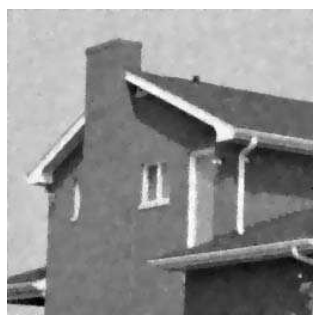

(j)

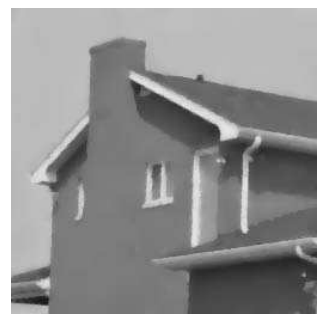

(n)

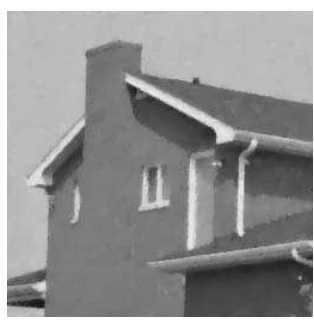

$(\mathrm{r})$

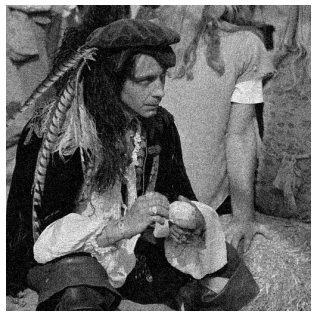

(c)

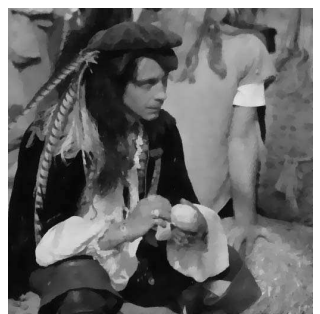

$(\mathrm{g})$

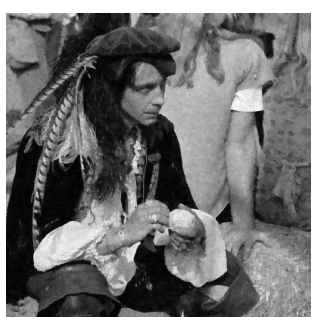

(k)

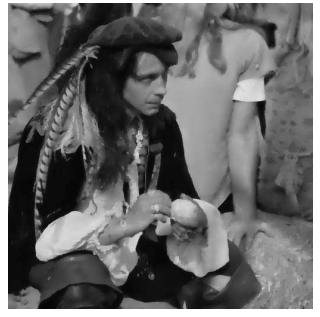

(o)

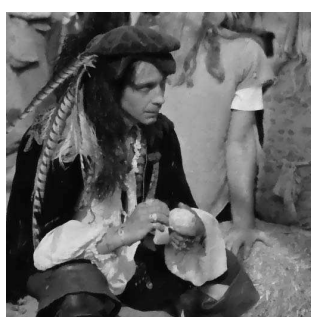

(s)

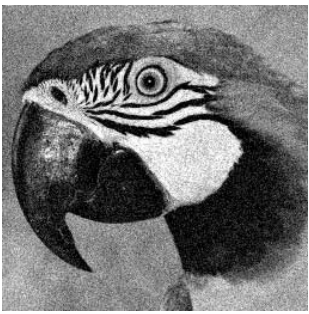

(d)

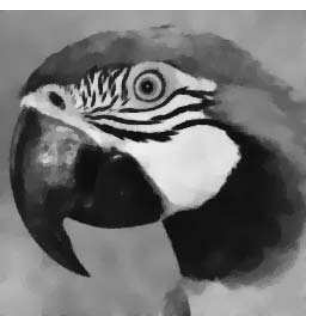

(h)

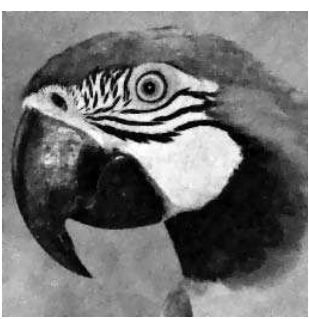

(1)

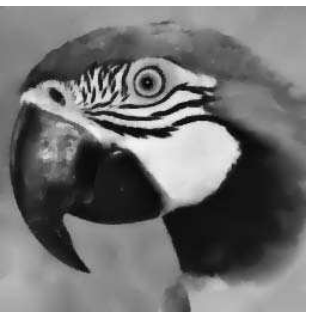

(p)

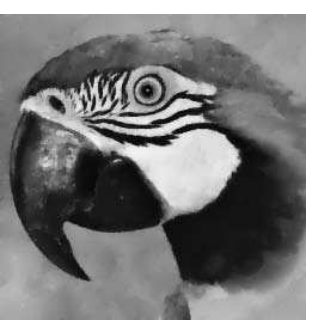

(t)

Figure 4: Gray scale image denoising results. (a)-(d): noisy images corrupted by random Gaussian noise $\sigma=20$, (e)-(h): denoised images by TV model, (i)-(I): TV boosting images, (m)-(p): TGV denoised images, (q)-(t): TGV boosting images. 


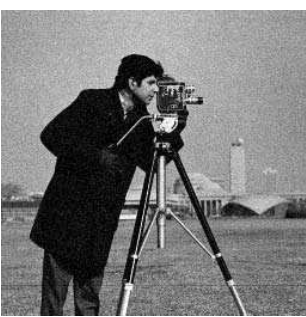

(a)

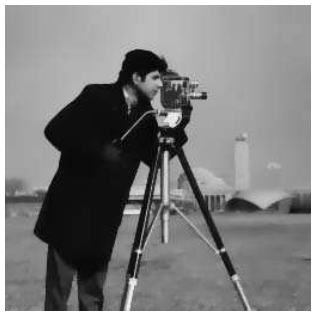

(e)

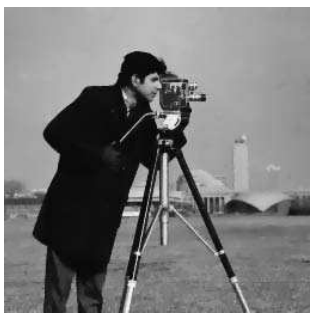

(i)

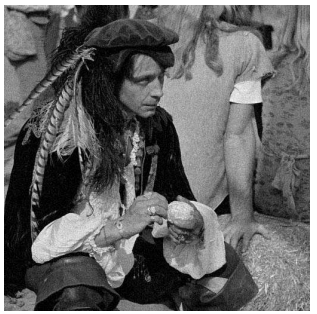

(b)

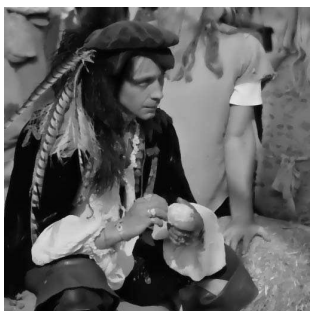

(f)

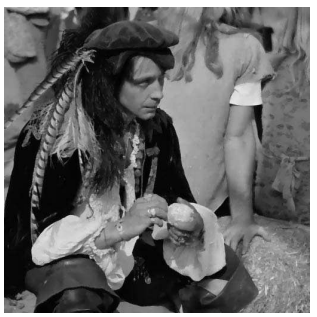

(j)

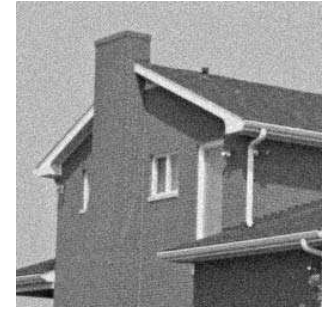

(c)

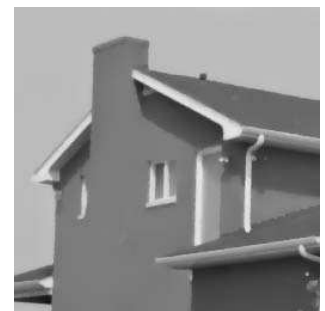

$(\mathrm{g})$

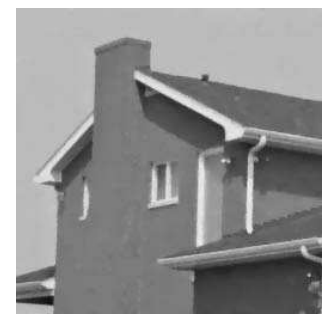

(k)

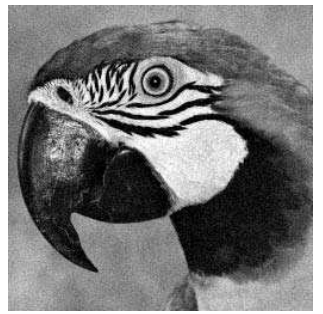

(d)

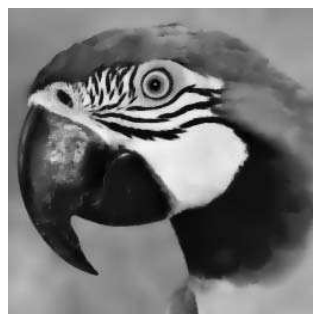

(h)

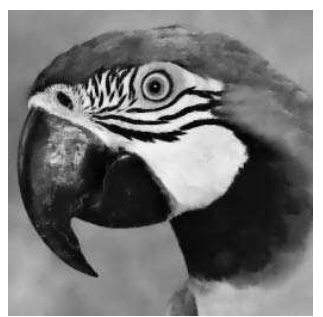

(1)

Figure 5: Gray scale image denoising results. (a)-(d): noisy images corrupted by random Gaussian noise $\sigma=10$, (e)-(h): denoised images by TGV model, (i)-(l): Boosting images by our method.

TGV-boost has best results and close match to the original image, see Figs. 3(e) and 3(f).

Further tests are done on the natural images, where we compare the denoising results by TV, TGV and results by our boosting method. We put restored results by TV and TGV model in Fig. 4, where one can observe that TV denoising results in the second row of Fig. 4 seem oversmoothed, while the results by our boosting method in the third row preserve more image structures. One can also observed that the denoised results by TGV in fourth row of Fig. 4 has better results than TV, while TGV-boost has best results, see last row in Fig. 4. Similarly, in Fig. 5 one can see the obvious improvement by our boosting procedure over TGV results. We perform the tests on different images with different noise level and put all the SNR values in Table 1.

\subsection{Image deblurring}

To generate different blurry images we use the Gaussian kernel with five different sizes $r \in\{4,6,8,10,12\}$ and Gaussian noise is added with fixed standard deviation $\sigma=5$. 


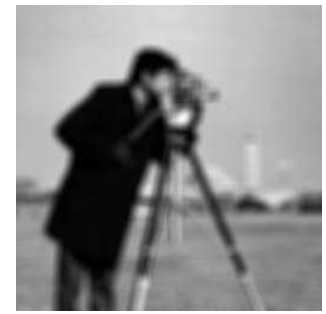

(a)

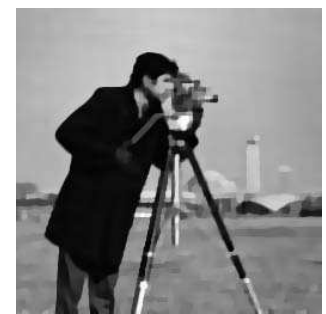

(e)

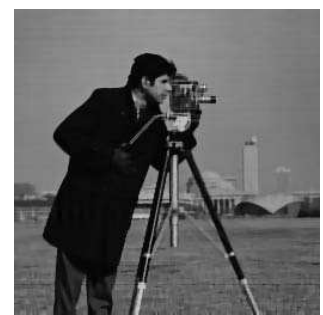

(i)

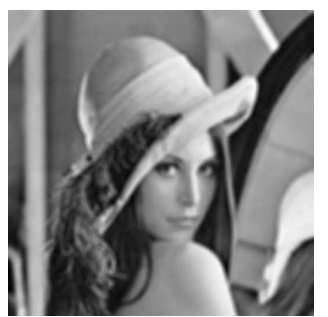

(b)

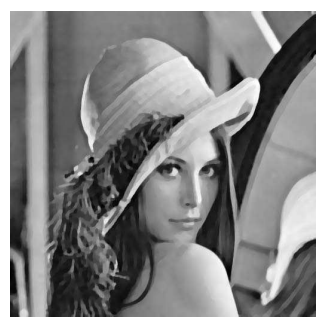

(f)

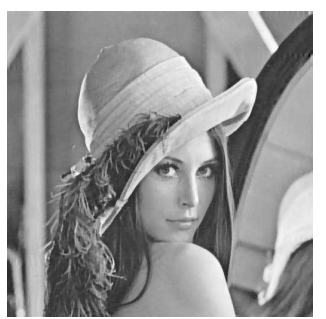

(j)

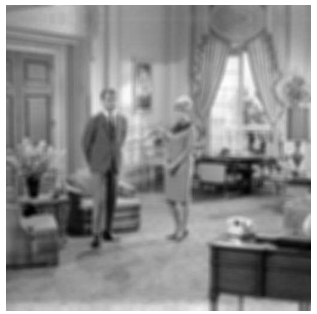

(c)

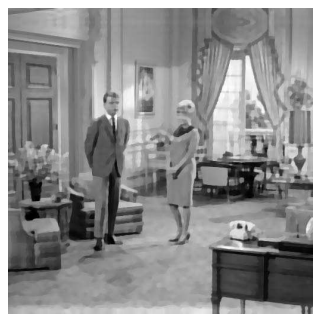

$(\mathrm{g})$

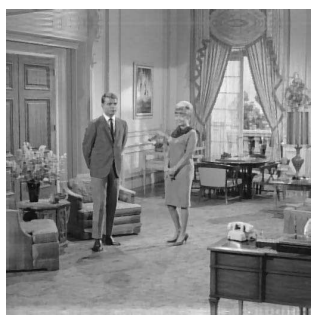

(k)

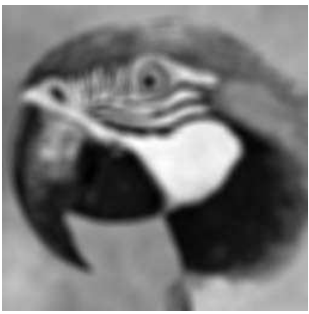

(d)

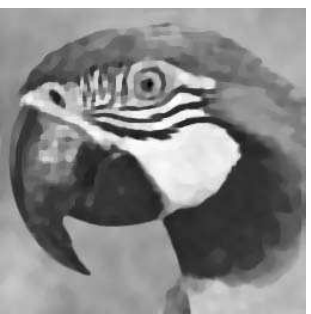

(h)

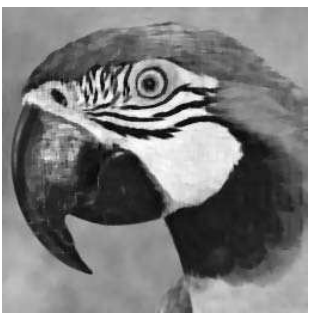

(1)

Figure 6: Gray scale image deblurring results. (a)-(d): blur images corrupted by Gaussian kernel of size $r=10$ and standard deviation $\sigma=5$, (e)-(h): deblurred images by TV model, (i)-(l): Boosting images by our method.

In [24], author used Brent's method [5] to find the optimal value of $\lambda$ with the minimum mean square error, and therefore initialize the iteration with the following empirical estimate of $\lambda$ as $\lambda=r\left(\frac{117.0}{\sigma}+\frac{4226.3}{\sigma^{2}}\right)$. In this experiment we fixed $\rho=0.0001$. We set $k=5$ as the outer iteration number and inner iterations are chosen the same as defaulted in the original package tvreg (available on image processing online(IPOL) [24]). In Table 2, we show the SNRs of our proposed method compared with TV algorithm, where we can see obvious increase. Comprehensive results are put in Fig. 6, which shows the results by our method are significantly improved visually.

\subsection{Image inpainting}

We perform the test on two types of inpainting task, i.e. missing region (see Fig. 7) and text inserting (see Fig. 8). Weak noise is added with standard deviation $\sigma=0.001$ for inpainting. In the first example in Fig. 7, for TV inpainting set $\lambda=20$. The proposed method is initialized with the same $\lambda$, and set $\tau=1.38, \rho=0.1$. Set the number of outer 


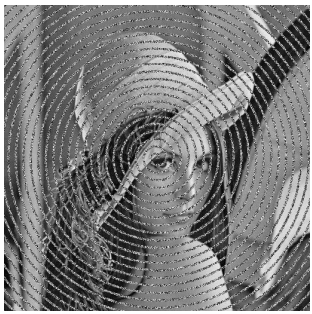

(a)

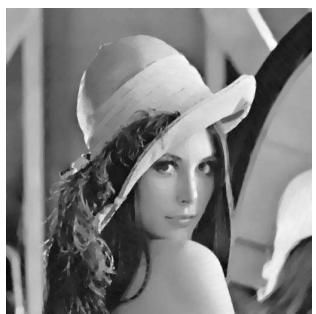

(e) TV SNR: $15.63 \mathrm{~dB}$

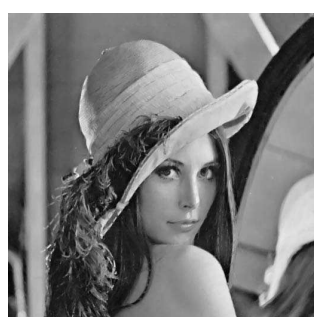

(i) Boosting SNR: $17.90 \mathrm{~dB}$

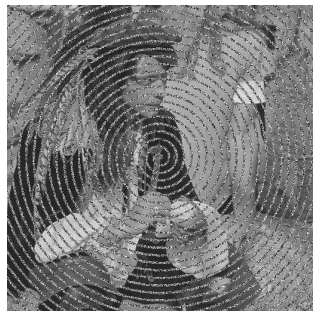

(b)

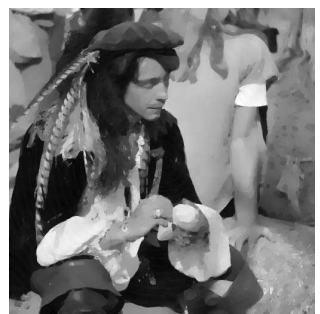

(f) TV SNR: $13.43 \mathrm{~dB}$

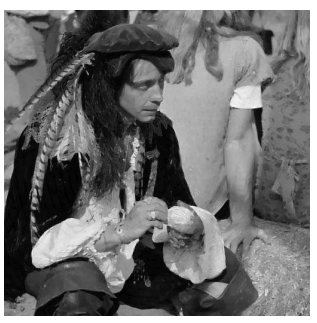

(j) Boosting SNR: $16.09 \mathrm{~dB}$

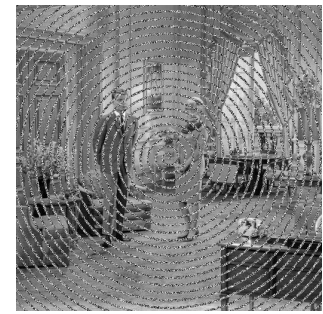

(c)

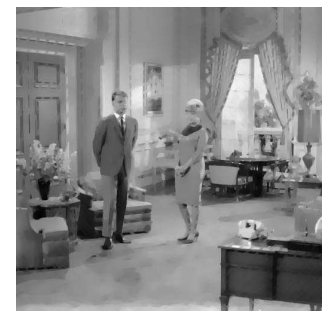

(g) TV SNR: $11.71 \mathrm{~dB}$

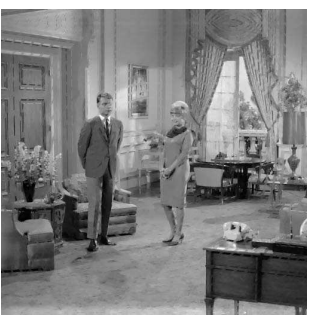

(k) Boosting SNR: $13.95 \mathrm{~dB}$

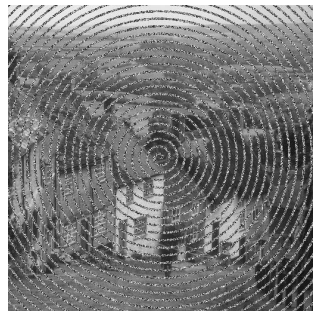

(d)

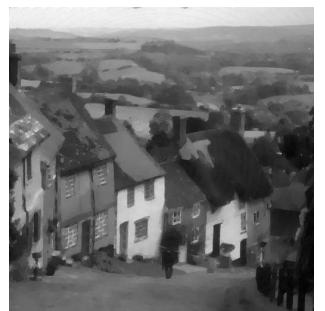

(h) TV SNR: $13.98 \mathrm{~dB}$

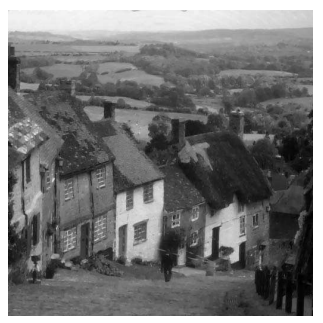

(1) Boosting SNR: $16.84 \mathrm{~dB}$

Figure 7: Gray scale image inpainting results. (a)-(d): images with missing region of level 6 and also corrupted by random Gaussian noise $\sigma=0.001,(\mathrm{e})-(\mathrm{h})$ : inpainted images by TV model, (i)-(l): Boosting images by our method.

loop as $k=6$. Similarly in the second example in Fig. 8 set $\lambda=30$ for TV algorithm, and $\rho=0.5, \tau=1.5, k=5$. In both two examples one can readily see the improvement by the proposed method.

Finally in Fig. 9 we show the histories of SNR with respect to $\lambda_{k}$ during the iterations for image restoration, which demonstrates that our proposed method is rather robust with respect to the iteration number.

\section{Conclusions}

In this paper we propose a new boosting framework for the popular variation models for image restorations, which can restore the degraded images with more structures compared with the classical TV models. On the other hand, the proposed method is simple for use and it can be extended to a wide range of restoration tasks. In the future we will consider to develop much more fast and efficient boosting scheme for variational problem 


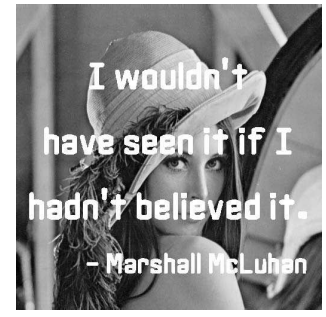

(a)

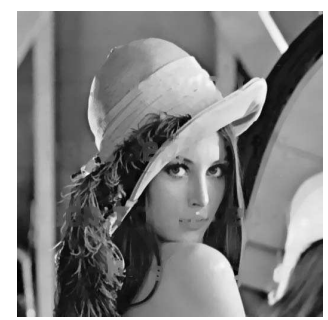

(e) TV SNR: $16.47 \mathrm{~dB}$

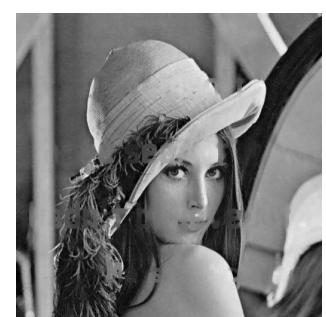

(i) Boosting $17.55 \mathrm{~dB}$

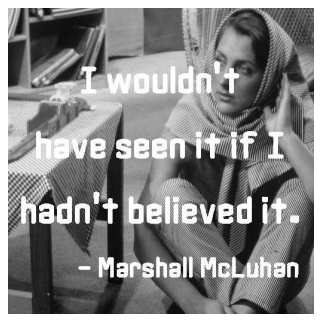

(b)

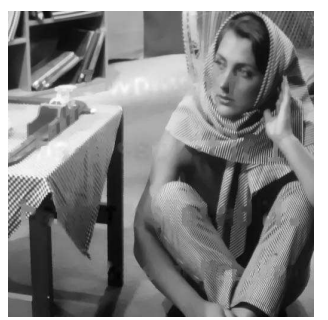

(f) TV SNR: $14.26 \mathrm{~dB}$

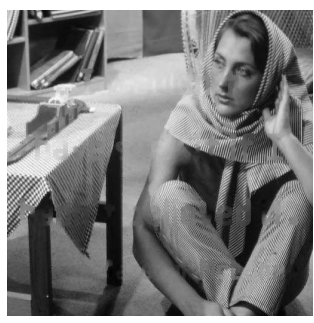

(j) Boosting SNR: $16.75 \mathrm{~dB}$

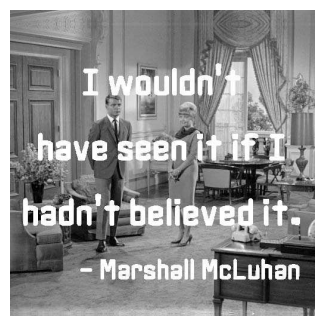

(c)

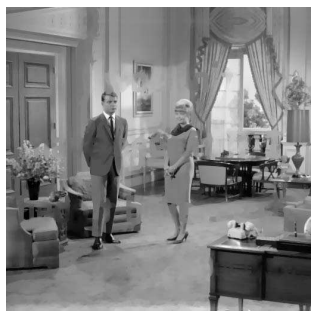

(g) TV SNR: $13.72 \mathrm{~dB}$

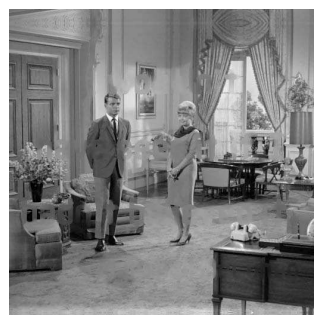

(k) Boosting SNR: $15.35 \mathrm{~dB}$

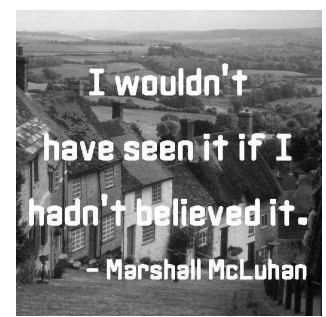

(d)

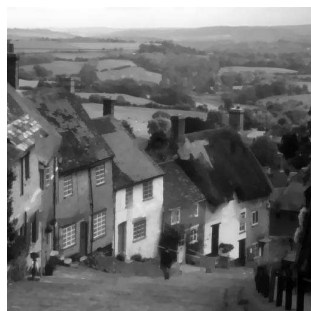

(h) TV SNR: $15.64 \mathrm{~dB}$

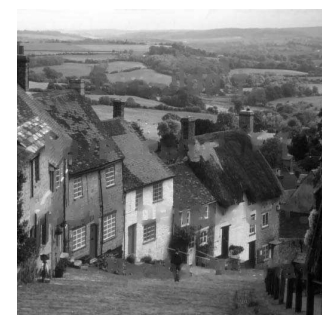

(1) Boosting SNR: $18.26 \mathrm{~dB}$

Figure 8: Gray scale image inpainting results. (a)-(d): images with text and also corrupted by random Gaussian noise $\sigma=0.001$, (e)-(h): inpainted images by TV model, (i)-(l): Boosting images by our method.

by exploiting fundamental properties of regularization parameter and group sparsity prior of the degraded images.

Acknowledgments The authors would like to thank Dr. Pascal Getreuer for his codes available online. The authors would like to thank the anonymous reviewers for their helpful and constructive comments. The work of Dr. C. Wu was supported by National Natural Science Foundation of China (Grant No. 11301289 and 11531013). Dr. H. Chang was partially supported by National Natural Science Foundation of China (Nos. 11501413 and 51609259), China Scholarship Council (CSC), Young backbone of innovative personnel training program No. 043-135205GC37, 2017-Outstanding Young Innovation Team Cultivation Program No. 043-135202TD1703, Innovation Project No. 043-135202XC1605 of Tianjin Normal University, and the Research Program of China Institute of Water Resources and Hydropower Research (Nos. JZ0145B472016 and JZ0145B862017). 


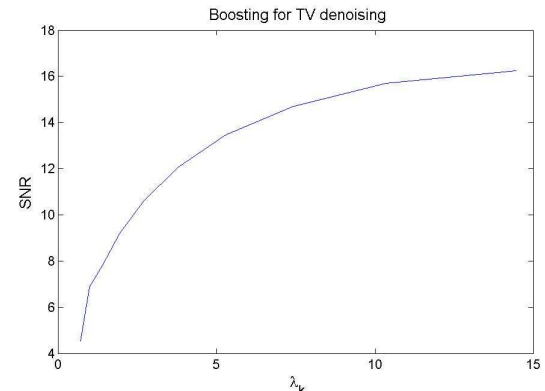

(a)

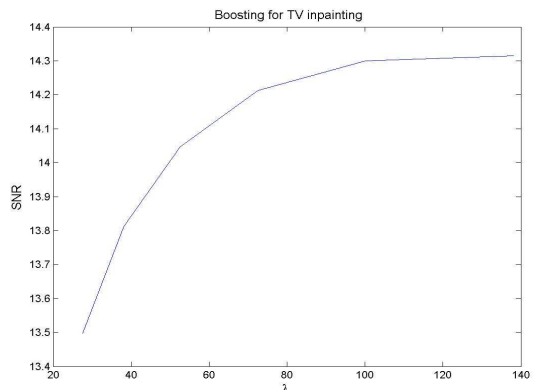

(c)

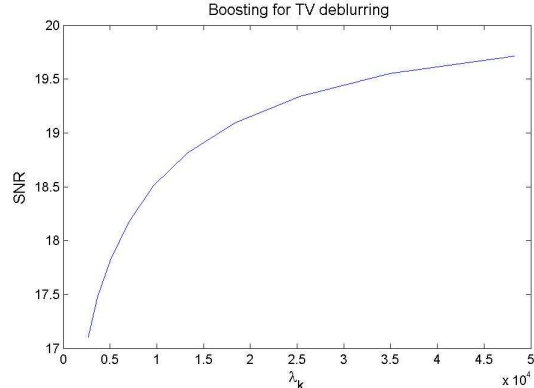

(b)

Figure 9: (a)-(c): Evaluation of $\lambda_{k}$ and SNR for House image (a): image denoising on noisy $\sigma=20$, (b): image deblurring on Gaussian kernel size $r=10$ and standard deviation $\sigma=5$, (c): image inpainting on $60 \%$ missing region plus noise with $\sigma=0.001$.

\section{References}

[1] P. ARIas, G. Facciolo, V. CASElles, AND G. SAPIro, A variational framework for exemplar-based image inpainting, Int. J. Comput. Vision, 93(3) (2011), pp. 319-347.

[2] J. F. Aujol, Some first-order algorithms for total variation based image restoration, J. Math. Imaging Vis., 34(3) (2009), pp. 307-327.

[3] K. BREDies AND M. Holler, Regularization of linear inverse problems with total generalized variation, J. Inverse Ill-pose. P., 22(6) (2014), pp. 871-913.

[4] K. BRedies, K. Kunisch AND T. Pock, Total generalized variation, SIAM Journal on Imaging Sciences, 3(3) (2010), pp. 492-526.

[5] R. P. BRent, Algorithms for minimization without derivatives, Math. Comput., 19(5) (1974).

[6] A. M. Bruckstein, D. L. Donoho and M. Elad, From sparse solutions of systems of equations to sparse modeling of signals and images, SIAM Rev., 51(1) (2009), pp. 34-81.

[7] A. BuAdes, B. Coll AND J. M. MoReL, A review of image denoising algorithms, with a new one, Multiscale Model. Sim., 4(2) (2005), pp. 490-530.

[8] A. Chambolle, An algorithm for total variation minimization and applications, J. Math. Imaging Vis., 20(1-2) (2004), pp. 89-97.

[9] A. Chambolle and P. L. Lions, Image recovery via total variation minimization and related problems, Numer. Math., 76(2) (1997), pp. 167-188.

[10] S. H. Chan, R. Khoshabeh, K. B. Gibson, P. E. Gill and T. Q. NGuYen, An augmented lagrangian 
method for total variation video restoration, IEEE T. Image Process., 20(11) (2011), pp. 30973111.

[11] T. Chan, S. Esedoglu, F. Park and A. Yip, Recent developments in total variation image restoration, Mathematical Models of Computer Vision, 17(2), 2005.

[12] T. Chan, A. MARquina AND P. Mulet, High-order total variation-based image restoration, SIAM J. Sci. Comput., 22(2) (2000), pp. 503-516.

[13] T. F. Chan, S. Esedoglu And F. PARK, A fourth order dual method for staircase reduction in texture extraction and image restoration problems, IEEE Image Proc., 2000, pp. 4137-4140.

[14] H. Chang, X. C. TAI, L. L. WANG AND D. YANG, Convergence rate of overlapping domain decomposition methods for the rudin-osher-fatemi model based on a dual formulation, SIAM Journal on Imaging Sciences, 8(1) (2015), pp. 564-591.

[15] H. Chang, X. Zhang, X. C. TAI AND D. YANG, Domain decomposition methods for nonlocal total variation image restoration, J. Sci. Comput., 60(1) (2014), pp. 79-100.

[16] M. R. Charest, M. Elad and P. Milanfar, A general iterative regularization framework for image denoising, Conference on IEEE, 2006, pp. 452-457.

[17] M. R. Charest and P. Milanfar, On iterative regularization and its application, IEEE T. Circ. Syst. Vid., 18(3) (2008), pp. 406-411.

[18] K. Dabov, A. Foi, V. Katkovnik and K. Egiazarian, Image denoising by sparse 3-d transformdomain collaborative filtering, IEEE T. Image Process., 16(8) (2007), pp. 2080-2095

[19] M. Elad, Prologue, Sparse and Redundant Representations, 2010, pp. 3-15.

[20] M. Elad AND M. AHARON, Image denoising via sparse and redundant representations over learned dictionaries, IEEE T. Image Process., 15(12) (2006), pp. 3736-3745.

[21] Y. Freund AND R. E. Schapire, A desicion-theoretic generalization of on-line learning and an application to boosting, Journal of Computer and System Sciences, 55(1) (1997), pp. 119139.

[22] N. P. Galatsanos and A. K. Katsaggelos, Methods for choosing the regularization parameter and estimating the noise variance in image restoration and their relation, IEEE T. Image Process., 1(3) (1992), pp. 322-336.

[23] P. Getreuer, Rudin-osher-fatemi total variation denoising using split bregman, Image Processing On Line, 2 (2012), pp. 74-95.

[24] P. Getreuer, Total variation deconvolution using split bregman, Image Processing On Line, 2 (2012), pp. 158-174.

[25] P. Getreuer, Total variation inpainting using split bregman, Image Processing On Line, 2 (2012), pp. 147-157.

[26] T. GolDSTEIN AND S. OsheR, The split bregman method for l1-regularized problems, SIAM Journal on Imaging Sciences, 2(2) (2009), pp. 323-343.

[27] M. R. Hestenes, Multiplier and gradient methods, J. Optimiz. Theory App, 4(5) (1969), pp. 303-320.

[28] W. Hinterberger and O. Scherzer, Variational methods on the space of functions of bounded hessian for convexification and denoising, Computing, 76(1) (2006), pp. 109-133.

[29] J. KAISER AND R. HAMming, Sharpening the response of a symmetric nonrecursive filter by multiple use of the same filter, IEEE T. Acoust. Speech, 25(5) (1977), pp. 415-422.

[30] M. LysakER, A. LundERVOLD AND X. C. TAI, Noise removal using fourth-order partial differential equation with applications to medical magnetic resonance images in space and time, IEEE T. Image Process., 12(12) (2003), pp. 1579-1590.

[31] M. LYSAKER AND X. C. TAI, Iterative image restoration combining total variation minimization and a second-order functional, Int. J. Comput. Vision, 66(1) (2006), pp. 5-18.

[32] Y. MEYER, Oscillating patterns in image processing and nonlinear evolution equations: the fif- 
teenth Dean Jacqueline B. Lewis memorial lectures, American Mathematical Soc., 22 (2001).

[33] M. NiкоLova, Minimizers of cost-functions involving nonsmooth data-fidelity terms. application to the processing of outliers, SIAM J. Numer. Anal., 40(3) (2002), pp. 965-994.

[34] M. Nikolova, A variational approach to remove outliers and impulse noise, J. Math. Imaging Vis., 20(1-2) (2004), pp. 99-120.

[35] S. Osher, M. Burger, D. Goldfarb, J. Xu AND W. Yin, An iterative regularization method for total variation-based image restoration, Multiscale Model. Sim., 4(2) (2005), pp. 460-489.

[36] M. J. Powell, A Method for Non-Linear Constraints in Minimization Problems, UKAEA, 1967.

[37] Y. RomAno AND M. ELAD, Boosting of image denoising algorithms, SIAM Journal on Imaging Sciences, 8(2) (2015), pp. 1187-1219.

[38] L. I. RUdin, S. OsheR AND E. FATEMI, Nonlinear total variation based noise removal algorithms, Physica D: Nonlinear Phenomena, 60(1) (1992), pp. 259-268.

[39] O. Scherzer, M. Grasmair, H. Grossauer, M. Haltmeier and F. Lenzen, Variational Methods in Imaging, Springer, 320 (2009).

[40] J. Shen And T. F. Chan, Mathematical models for local nontexture inpaintings, SIAM J. Appl. Math., 62(3) (2002), pp. 1019-1043.

[41] J. Shen, S. H. KANG AND T. F. ChAN, Euler's elastica and curvature-based inpainting, SIAM J. Appl. Math., 63(2) (2003), pp. 564-592.

[42] L. Sun AND K. Chenz, A new iterative algorithm for mean curvature-based variational image denoising, BIT Numerical Mathematics, 54(2) (2014), pp. 523-553.

[43] E. TAdMOR, S. NezZAR AND L. Vese, A multiscale image representation using hierarchical (bv, $l$ 2) decompositions, Multiscale Model. Sim., 2(4) (2004), pp. 554-579.

[44] X. C. TAI, J. HAHn AND G. J. Chung, A fast algorithm for euler's elastica model using augmented lagrangian method, SIAM Journal on Imaging Sciences, 4(1) (2011), pp. 313-344.

[45] X. C. TAI AND C. WU, Augmented lagrangian method, dual methods and split bregman iteration for rof model, International Conference on Scale Space and Variational Methods in Computer Vision, Springer, 2009, pp. 502-513.

[46] H. TAlebi, X. Zhu and P. Milanfar, How to saif-ly boost denoising performance, IEEE T. Image Process., 22(4) (2013), pp. 1470-1485.

[47] J. W. TukeY, Exploratory Data Analysis, 1977.

[48] Y. WANG, J. YANG, W. YIN AND Y. ZHANG, A new alternating minimization algorithm for total variation image reconstruction, SIAM Journal on Imaging Sciences, 1(3) (2008), pp. 248-272.

[49] C. WU AND X. C. TAI, Augmented lagrangian method, dual methods, and split bregman iteration for rof, vectorial tv, and high order models, SIAM Journal on Imaging Sciences, 3(3) (2010), pp. 300-339.

[50] C. Wu, J. Zhang And X. C. TAI, Augmented lagrangian method for total variation restoration with non-quadratic fidelity, Inverse Probl. Imag., 5(1) (2011), pp. 237-261.

[51] J. YANG, W. YIN, Y. ZHANG AND Y. WANG, A fast algorithm for edge-preserving variational multichannel image restoration, SIAM Journal on Imaging Sciences, 2(2) (2009), pp. 569-592.

[52] J. Yang, Y. Zhang And W. Yin, An efficient tvl1 algorithm for deblurring multichannel images corrupted by impulsive noise, SIAM J. Sci. Comput., 31(4) (2009), pp. 2842-2865.

[53] Y. L. You AND M. KAvEH, Fourth-order partial differential equations for noise removal, IEEE T. Image Process., 9(10) (2000), pp. 1723-1730.

[54] J. ZHANG AND K. CHEN $A$ total fractional-order variation model for image restoration with nonhomogeneous boundary conditions and its numerical solution, SIAM Journal on Imaging Sciences, 8(4) (2015), pp. 2487-2518.

[55] D. ZORAN AND Y. WEISs, From learning models of natural image patches to whole image restoration, IEEE International Conference on Computer Vision, 2011, pp. 479-486. 